\title{
Herpetofauna de Tacámbaro, Michoacán, México
}

\section{Herpetofauna from Tacámbaro, Michoacán, México}

\author{
Oscar Medina-Aguilar, Javier Alvarado-Díaz e Ireri Suazo-Ortuño ${ }^{\bowtie}$ \\ Instituto de Investigaciones sobre los Recursos Naturales, Universidad Michoacana de San Nicolás de Hidalgo. Av. San Juanito Itzicuaro s/n Col. Nva. \\ Esperanza, 58337 Morelia, Michoacán, México. \\ 凶ireri.suazo@gmail.com
}

\begin{abstract}
Resumen. Se presentan los resultados de un inventario herpetofaunístico realizado en 2007 y 2008 en 6 tipos de vegetación del municipio de Tacámbaro, Michoacán. Se registró un total de 21 especies de anfibios (8 familias) y 58 especies de reptiles (16 familias). Los estimadores de riqueza indican que se registraron entre el 85 y $91 \%$ de las especies de anfibios esperadas; entre 77 y $85 \%$ de las especies de lagartijas y entre 41 y $78 \%$ de las especies de serpientes. En la selva baja caducifolia se registraron 43 especies de anfibios y reptiles, 29 en el bosque espinoso, 17 en el bosque de pino-encino, 13 en el bosque de encino, 12 en el bosque de pino y 7 en el bosque de oyamel. De 79 especies registradas en el municipio, 49 (62\%) son endémicas de México y 3 del estado de Michoacán. De las especies registradas, 37 (47\%) se encuentran en la lista de la Norma Oficial Mexicana en alguna categoría de riesgo. La serpiente Leptotyphlops maximus se registra por primera vez para el estado de Michoacán.
\end{abstract}

Palabras clave: anfibios, reptiles, Tacámbaro, Michoacán.

\begin{abstract}
A herpetofaunistic survey was carried out in 2007 and 2008 in 6 vegetation types in the Municipality of Tacámbaro, state of Michoacán, México. A total of 21 amphibian species ( 8 families) and 58 reptile species (16 families) were registered. Species richness estimators indicated that from 85 to $91 \%$ of amphibian species, 77 to $85 \%$ of lizard species and 41 to $78 \%$ of snake species were registered. Forty-three species were registered in tropical deciduous forest, 29 in thorn forest, 17 in pine-oak forest, 13 in oak forest, 12 in pine forest, and 7 in fir forest. Of 79 species registered, 49 (62\%) are endemic to México and 3 species are endemic to Michoacán. Thirty-seven (47\%) species are listed in the Norma Oficial Mexicana of threatened species. The snake Leptotyphlops maximus was registered for the first time in Michoacán.
\end{abstract}

Key words: amphibians, reptiles, Tacámbaro, Michoacán

\section{Introducción}

La herpetofauna es uno de los componentes de la biodiversidad mexicana que tiene especial relevancia por sus altos valores de riqueza (aproximadamente 361 especies de anfibios y 804 de reptiles; Flores-Villela y CansecoMárquez, 2004) y endemismo (48 \% de las especies de anfibios y $46 \%$ de las de reptiles son exclusivas de México; Mittermeier et al., 1997; AmphibiaWeb, 2007; CONABIO, 2008).

En Michoacán, un estado con una fisiografía definida principalmente por el Eje Volcánico Transmexicano (EVT), la sierra Madre del Sur y la depresión del Balsas, se han registrado 53 especies de anfibios y 138 especies de reptiles (CONABIO, 2008). A pesar de que se han realizado diversos estudios herpetofaunísticos (e.g., Schmidt y Shannon, 1947; Peters, 1954; Duellman, 1961, 1965) aún existen vacíos de información sobre la diversidad de

Recibido: 17 agosto 2010; aceptado: 11 mayo 2011 anfibios y reptiles en algunas regiones del estado, especialmente de la sierra Madre del Sur y las estribaciones sur-occidentales del EVT que conectan este último con la depresión del Balsas. Una de estas regiones es la correspondiente al municipio de Tacámbaro con parte de su territorio ubicado en las estribaciones sureñas del EVT y parte en la depresión del Balsas. En el estudio sobre la diversidad herpetofaunística de Michoacán, Duellman (1961) registró solamente una especie de lagartija (Anolis nebulosus) y una de serpiente (Pituophis deppei) en localidades del municipio de Tacámbaro.

El conocimiento sobre la composición y distribución de la herpetofauna es una herramienta esencial para la planificación de estrategias y acciones de conservación, especialmente si se consideran la extinción y el declive global de las especies y poblaciones de anfibios y reptiles (Gibbons et al., 2000; Mendelson et al., 2006; Bancroft et al., 2008), por lo que este trabajo tiene como objetivo dar a conocer la composición y riqueza de los anfibios y reptiles del municipio de Tacámbaro, Michoacán. 


\section{Materiales y métodos}

La fisiografía del territorio del municipio de Tacámbaro, Michoacán $\left(19^{\circ} 25^{\prime} \mathrm{N}, 19^{\circ} 06^{\prime} \mathrm{N}\right.$ y $101^{\circ} 19^{\prime} \mathrm{O}$, 101³8' O; Fig. 1) está determinada por el EVT, las estribaciones sureñas del EVT y la depresión del Balsas. La elevación del terreno varía entre los 800 y 2900 m snm. La temperatura media anual es de $18^{\circ} \mathrm{C}$, con una precipitación pluvial anual de $1451.6 \mathrm{~mm}$, de la cual $80 \%$ se concentra entre julio y octubre, después de 7 meses de estiaje (INEGI, 1985). Los tipos de vegetación con mayor extensión en el municipio, en orden descendiente son: bosque mixto de pino-encino, selva baja caducifolia, bosque de pino, bosque espinoso, bosque de encino y bosque de oyamel (INEGI, 1985). Las actividades humanas en el área han moldeado un paisaje que en la actualidad se encuentra dominado por huertas de aguacate en las estribaciones del Eje Volcánico Transmexicano y por caña de azúcar en la depresión del Balsas, entremezclados con otros cultivos, como maíz, trigo, frijol, naranja, alfalfa, sorgo, garbanzo, mango y durazno (INEGI, 1985).

Para el muestreo de herpetofauna se realizaron 15 visitas al área de estudio durante 2007 en los meses de marzo, abril, mayo, junio y diciembre (temporada de estiaje) y julio, agosto, septiembre y octubre (temporada de lluvias). En 2008 se efectuaron 3 visitas adicionales en los meses de abril y junio. Cada vista fue de 3 días para un total de 54 días de trabajo de campo.

Se muestrearon los 6 diferentes tipos de vegetación predominantes en el municipio: selva baja caducifolia, bosque espinoso, bosque de encino, bosque de pino-encino, bosque de pino y bosque de oyamel para registrar las especies de anfibios y reptiles mediante la técnica de búsqueda por encuentro visual con tiempo definido (Lips et al., 2001). El esfuerzo de muestreo se registró en horas/persona. Los muestreos fueron diurnos (09:00-13:00 h) y nocturnos (19:00-23:00 h). El esfuerzo de muestreo fue diferente en cada tipo de vegetación (bosque de pino 46.66 horas/persona, bosque de oyamel 47.75 horas/persona, bosque de encino 53.54 horas/persona, bosque espinoso 53.98 horas/ persona, bosque de pino-encino 62.45 horas/persona y selva baja caducifolia 153.69 horas/persona).

Para el tipo de hábito (sustrato en el que desarrollan sus actividades) de las especies se siguieron los criterios de García y Ceballos (1994) y Savage (2002). Para complementar el inventario, también se consideraron observaciones esporádicas, registros fuera de los tiempos de muestreo, ejemplares atropellados en las carreteras y aquellos proporcionados por los habitantes locales. Cuando surgieron dudas sobre la correcta identificación durante el trabajo de campo, los ejemplares fueron colectados y transportados al Laboratorio de Herpetolo- gía del Instituto de Investigaciones sobre los Recursos Naturales (INIRENA) de la Universidad Michoacana de San Nicolás de Hidalgo para una adecuada identificación, mediante publicaciones y claves taxonómicas de Smith y Taylor (1945, 1948, 1950), Duellman (1961, 1965, 2001), Casas y McCoy (1979), Ramírez-Bautista (1994), Flores-Villela et al. (1995) y Huacuz (1995). Para la actualización taxonómica de los nombres científicos se revisaron los trabajos de Flores-Villela y CansecoMárquez (2004) y Faivovich et al. (2005). Se depositaron ejemplares voucher en la colección herpetológica del INIRENA. El número de ejemplares recolectados no fue mayor de 2 por especie (permiso de colecta científica DGVS/FAUT-0113).

Para evaluar la representatividad del muestreo se utilizaron 3 estimadores no paramétricos: ICE, Chao 2 y bootstrap siguiendo los criterios de Magurran (2004) y empleando el programa Estimates 7.0 (Colwell, 2005). Estos índices no paramétricos estiman el número de especies que faltan por registrar, basándose en la cuantificación de la rareza de las especies colectadas. Los estimadores ICE y Chao 2 son predictores confiables de la riqueza de especies cuando se tienen muestras pequeñas y el bootstrap tiene resultados más precisos al estimar la riqueza de ensamblajes con gran cantidad de especies raras (Magurran, 2004). Para evaluar diferencias entre el número de especies observadas y el número de especies estimadas se llevó a cabo un análisis de chi cuadrada utilizando la corrección de Yates (Sokal y Rohlf, 1995). Con base en los valores máximos de riqueza estimada se determinó el porcentaje de especies registradas durante el presente estudio (completeness de Soberón y Llorente, 1993). El estado de conservación de las especies registradas se determinó siguiendo la Norma Oficial Mexicana (NOM059-SEMARNAT-2001; SEMARNAT, 2002). La base de datos de los anfibios y reptiles recolectados y la información curatorial de este acervo forman parte de la colección herpetológica del INIRENA y están disponibles para su consulta en: (http://www.inirena.umich.mx).

\section{Resultados}

Los estimadores ICE y Chao 2 llegaron a la asíntota más rápidamente que la riqueza observada y el bootstrap. Este último presentó un patrón similar al de la riqueza observada (Fig. 2). En general, los estimadores reflejaron una mayor riqueza que la observada pero el análisis de $\mathrm{X}^{2}$ mostró que únicamente en el caso de las serpientes los estimadores ICE y Chao 2 estimaron significativamente más especies que las especies registradas en este estudio (Cuadro 1). En este estudio se logró representar entre el 77 y $91 \%$ de la riqueza para el caso de anfibios y lagartijas. 
Cuadro 1. Completitud del inventario, riqueza observada y riqueza esperada de anfibios y reptiles en el municipio de Tacámbaro Michoacán, México

\begin{tabular}{rlcccc}
\hline Grupo & \multicolumn{1}{c}{ Estimador } & Completitud (\%) & $\begin{array}{c}\text { Especies } \\
\text { observadas }\end{array}$ & Especies estimadas & $X^{2}$ \\
Anfibios & ICE & 85 & 21 & 25 & 0.2 \\
& Chao 2 & 91 & 21 & 33 & 2.3 \\
\multirow{5}{*}{ Lagartijas } & Bootstraps & 87 & 21 & 24 & 0.1 \\
& ICE & 77 & 23 & 30 & 0.6 \\
\multirow{5}{*}{ Serpientes } & Chao 2 & 80 & 23 & 29 & 0.4 \\
& Bootstraps & 85 & 33 & 81 & 0.2 \\
& ICE & 41 & 33 & 86 & $19.1^{*}$ \\
& Chao 2 & 50 & 33 & 43 & $22.5^{*}$ \\
\hline
\end{tabular}

$*=p<0.0001$

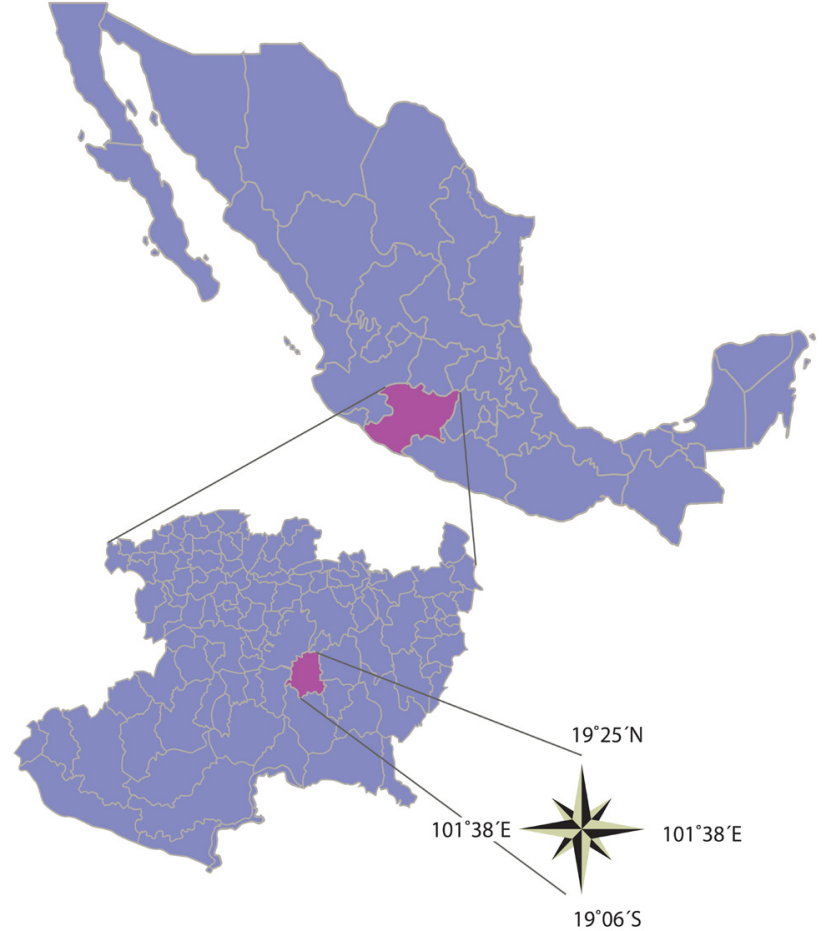

Figura 1. Ubicación del municipio de Tacámbaro, Michoacán, México.

Los estimadores Chao 2 y ICE tendieron a sobreestimar la riqueza de serpientes por más del doble, mientras que el bootstrap presentó un patrón muy similar al de la riqueza observada (Fig. 2). Basándose en los 2 primeros estimadores, se representó entre el 41 y $50 \%$ de la riqueza de serpientes, mientras que basándose en el bootstrap la riqueza representada fue de 78\% (Cuadro 1). El análisis de $\mathrm{X}^{2}$ mostró que no existen diferencias significativas entre las especies observadas y las estimadas en anfibios y lagartijas. En el caso de las serpientes, solamente el estimador bootstrap no fue diferente (Cuadro 1).

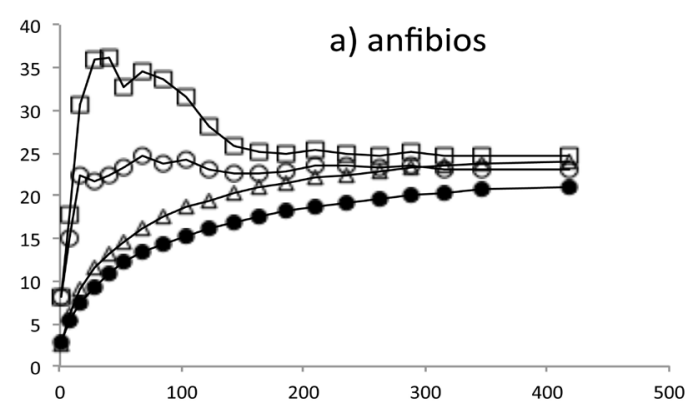

b) lagartijas

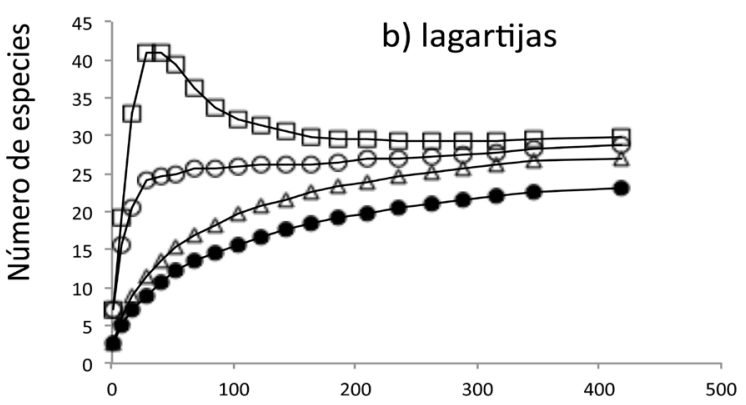

b) serpientes

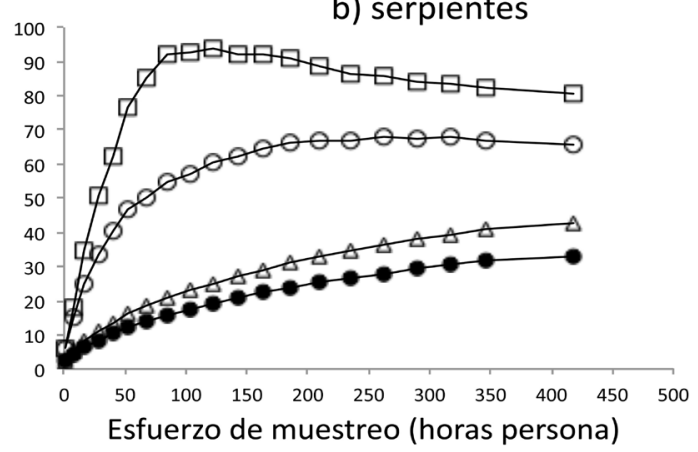

Figura 2. Curvas de acumulación de especies de anfibios, lagartijas y serpientes para el municipio de Tacámbaro, Michoacán. Especies observadas, ; estimadores de riqueza no paramétricos (ICE, $\square$, Chao 2,O y bootstrap, $\triangle$ ). 
Especies por tipo de vegetación. Se registraron 406 individuos pertenecientes a 21 especies de anfibios y 58 de reptiles, ubicados en 2 órdenes, 9 familias y 15 géneros de anfibios y 2 órdenes 15 familias y 40 géneros de reptiles (Apéndice 1). La herpetofauna del municipio representó el $41.14 \%$ del total registrado para el estado de Michoacán (191 especies, CONABIO, 2008).

La familia de anfibios que presentó mayor riqueza fue Hylidae, con 8 especies (38\% del total de la anfibiofauna registrada), seguida por la familia Leptodactilidae y Bufonidae con 3 especies cada una (14\%). El mayor número de reptiles, 26 especies, lo presentó la familia Colubridae, seguida por la familia Phrynosomatidae con 10 especies, lo que representa el 44.8 y $17.2 \%$ respectivamente del total de los reptiles registrados para el municipio.

En los tipos de vegetación predominantes en el municipio de Tacámbaro, la selva baja caducifolia presentó 43 especies, el bosque espinoso 29 , el bosque de pino-encino 17 , el bosque de encino 13, el bosque de pino 12 y el bosque de oyamel 7 especies (Apéndice 1).

De los anfibios, 9 especies fueron de un solo tipo de hábito: 4 acuático y 5 terrestre, y 12 presentaron más de un tipo de hábito. De las lagartijas, 15 especies fueron de un sólo hábito: 9 terrestre, 3 arborícola, 2 saxícola y 1 ripario, y 8 especies con más de un tipo de hábito. De las serpientes, 29 especies tienen un solo tipo de hábito: 22 terrestre, 4 fosorial y 3 arborícola; 4 especies presentaron más de un tipo de hábito. Las 2 especies de tortuga fueron de hábito acuático.

De las 79 especies registradas para el municipio de Tacámbaro, 33 (42\%, 7 especies de anfibios y 26 de reptiles) se encuentran incluidas en alguna categoría de riesgo en la NOM-059-SEMARNAT-2001: 25 especies están sujetas a protección especial y 12 amenazadas (Apéndice 1). También están incluidas en la lista de la NOM-059SEMARNAT-2001 15 especies registradas en la selva baja caducifolia, 11 en el bosque espinoso, 7 en el bosque de pino-encino, 6 en el bosque de encino y 5 en el bosque de pino y 5 en el bosque de oyamel.

Del total de especies registradas para el municipio, 48 (61\%) son endémicas de México. En la selva baja caducifolia se registraron 29 especies endémicas, seguidas en número por el bosque espinoso con 18 especies, el bosque de pino-encino con 12 especies, el bosque de pino con 10 especies, y los bosques de oyamel y encino con 5 especies cada uno. Se registraron 3 endémicas del estado de Michoacán; Ambystoma ordinarium exclusiva del EVT en bosque de pino y bosque de pino-encino; Mesoscincus altamirani y Leptotyphlops bressoni exclusivas de la depresión del Balsas, en selva baja caducifolia. Y por primera vez para el estado de Michoacán, se registró Leptotyphlops maximus, en selva baja caducifolia de la porción sur del municipio (Apéndice 1).

\section{Discusión}

Los resultados indican una riqueza importante (79 especies) de anfibios y reptiles en el municipio de Tacámbaro, especialmente si se compara con la riqueza registrada en estudios similares de otros municipios de Michoacán o en estudios realizados en regiones con áreas superiores a la de Tacámbaro.

Para el municipio de Tancítaro se registran 39 especies de anfibios y reptiles (Estrada-Virgen y Alvarado-Díaz, 2003), 40 especies para el de Morelia (García, 2002), 63 para el de Nuevo Urecho (González-Hernández y GarzaCastro, 2006) y 47 para el de Huetamo (Reyna, 2010). En cuanto a regiones, se han registrado 60 especies de anfibios y reptiles para la costa y sierra de Coalcomán (Peters, 1954) y 66 para el Playón de Mexiquillo (Vargas-Santa María y Flores-Villela, 2006).

En estados como Aguascalientes y Tlaxcala que poseen superficies mayores a las del municipio de Tacámbaro (5 $589 \mathrm{~km}^{2}$ y $4072 \mathrm{~km}^{2}$ respectivamente, $v s .787 .15 \mathrm{~km}^{2}$ de Tacámbaro), el número de especies es menor que el de las registradas en este estudio (71 especies en Aguascalientes, Vázquez y Quintero, 2005; 55 en Tlaxcala, Fernández et al., 2006).

Aunque estos valores de riqueza de herpetozoos pueden estar influenciados por el esfuerzo de captura aplicado (tiempo, número de sitios y método de muestreo) es evidente que el municipio de Tacámbaro presenta una alta riqueza de especies de anfibios y reptiles, lo que quizá se deba a que en la zona de estudio convergen 2 grandes regiones fisiográficas: el EVT y la depresión del Balsas. Por otro lado, la presencia de 6 diferentes tipos de vegetación en una superficie relativamente pequeña con especies exclusivas a cada tipo de vegetación refuerza la idea de que la diversidad de especies de un área es el resultado del marcado recambio de especies entre sitios (Rodríguez et al., 2003).

Previo a este trabajo, en el municipio de Tacámbaro sólo estaban registradas 3 especies de anfibios y reptiles (Anolis nebulosus, Pituophis deppei; Duellman, 1961 y Ambystoma ordinarium; Ortega, 2006); el presente estudio contribuye con 76 nuevos registros para el municipio. La herpetofauna de Tacámbaro (79 especies) representa $41.14 \%$ del total registrado para el estado de Michoacán (191 especies, CONABIO, 2008) y 6.78\% del total registrado para México (1 165 especies, Flores-Villela y Canseco-Márquez, 2004). Las curvas de acumulación de especies indicaron que el ensamble de anfibios y lagartijas fueron los mejor representados en este estudio, alcanzando niveles adecuados de representatividad. Se registraron 5 especies venenosas en el área de estudio: 3 serpientes de cascabel Crotalus molossus, Crotalus triseriatus y Cro- 
talus simus, así como el vipérido Agkistrodon bilineatus y la lagartija Heloderma horridum. El registro de estas especies es relevante porque representan un peligro para el ser humano. De importancia ecológica son Boa constrictor, Masticophis mentovarius y Pituophis lineaticolis, por mencionar algunas, ya que se les considera controladores biológicos por ser depredadoras de pequeños mamíferos, principalmente ratones y ratas; estos últimos son un riesgo para la salud pública, ya que pueden transmitir enfermedades, además de provocar disminución en el rendimiento agrícola.

El tipo de vegetación que presentó mayor riqueza de anfibios y reptiles fue la selva baja caducifolia, a pesar de que ocupa el segundo lugar en cuanto a superficie dentro del municipio; probablemente este resultado se deba a que la selva baja caducifolia del municipio de Tacámbaro forma parte de la depresión del Balsas, que se considera uno de los sitios con mayor riqueza y endemismos de México (Ochoa-Ochoa y Flores-Villela, 2006; García et al., 2007). De las especies registradas, $62 \%$ son endémicas de México y casi $50 \%$ se encuentran en alguna categoría de protección, lo que indica la urgente necesidad de establecer trabajos de monitoreo que permitan reconocer las amenazas y establecer estrategias de conservación.

\section{Agradecimientos}

Agradecemos el apoyo de José Gil Paz Gutiérrez, Jonatan Torres Coeto, Carlos Soto Rojas, Alejandra Sánchez y Ricardo Valencia Vargas por su apoyo en el trabajo de campo. El proyecto fue financiado por la Coordinación de la Investigación Científica de la UMSNH.

\section{Literatura citada}

AmphibiaWeb: Information on amphibian biology and conservation. [web application]. 2010. Berkeley, California: AmphibiaWeb. http://amphibiaweb.org/; última consulta 2.VII. 2010.

Bancroft, B. A., N. J. Baker y A. R. Blaustein. 2008. A metaanalysis of the effects of ultraviolet $\mathrm{B}$ radiation and its synergistic interactions with $\mathrm{pH}$, contaminants, and disease on amphibian survival. Conservation Biology 22:987-996.

Casas, A. G. y C. J. McCoy. 1979. Anfibios y reptiles de México, Claves ilustradas para su identificación. Limusa, México, D. F. 87 p.

Colwell, R. K. 2005. EstimateS: statistical estimation of species richness and shared species from samples. Version 7.5. user's guide and application. University of Connecticut, Storrs. http://purl.oclc.org/estimates; última consulta: 20.VII.2009.

CONABIO (Comisión Nacional para el Conocimiento y Uso de la Biodiversidad). 2008. Datos de distribución estatal del
Sistema Nacional de Información sobre Biodiversidad para Anfibios y Reptiles. CONABIO, México, D. F., México.

Duellman, E. W. 1961. The amphibians and reptiles of Michoacán México. University of Kansas Publications. Museum of Natural History 15:1-148.

Duellman, E. W. 1965. A biographic account of herpetofauna of Michoacán, México. University of Kansas Publications. Museum of Natural History 15:627-709.

Duellman, W. E. 2001. The Hylid frogs of Middle America. Society for the study of amphibians and reptiles, Ithaca. 1158 p.

Estrada-Virgen, A. y J. Alvarado-Díaz. 2003. Distribución y conservación de los anfibios y reptiles del Municipio de Tancítaro, Michoacán, México. Revista Ciencia Nicolaita 36:29-42.

Faivovich, J., C. F. B. Haddad, P. C. A. García, D. R. Frost, J. A. Campbell y W. C. Wheeler. 2005. Systematic review of the frog family Hylidae, with special reference to Hylinae: Phylogenetic analysis and taxonomic revision. Bulletin of the American Museum of Natural History 294:240.

Fernández, J. A., O. Sánchez y O. Flores-Villela. 2006. Anfibios y reptiles del Estado de Tlaxcala. In Inventarios herpetofaunísticos de México: avances en el conocimiento de su biodiversidad, A. Ramírez-Bautista, L. Canseco-Márquez y F. Mendoza-Quijano (eds.). Publicaciones de la Sociedad Herpetológica Mexicana 3:224-240.

Flores-Villela, O. y L. Canseco-Márquez. 2004. Nuevas especies y cambios taxonómicos para la herpetofauna mexicana. Acta Zoológica Mexicana 20:115-144.

Flores-Villela, O., Q. F. Mendoza, y G. González. 1995. Recopilación de claves para la determinación de anfibios y reptiles de México. Publicación Especial 10. Museo de Zoología, Facultad de Ciencias, Universidad Nacional Autónoma de México, México, D. F. 285 p.

García, A. y G. Ceballos. 1994. Guía de campo de los reptiles y anfibios de la costa de Jalisco, México. Fundación Ecológica de Cuixmala/ Instituto de Biología, UNAM, México D. F. $184 \mathrm{p}$.

García, A., H. Solano-Rodríguez y O. Flores-Villela. 2007. Patterns of alpha, beta and gamma diversity of the herpetofauna in Mexico's Pacific lowlands and adjacent interior valleys. Animal Biodiversity and Conservation 30.2:169-177.

García, P. D. 2002. Catálogo de anfibios y reptiles del municipio de Morelia, Michoacán, México. Tesis. Facultad de Biología, Universidad Michoacana de San Nicolás de Hidalgo, Morelia, Michoacán. 112 p.

Gibbons, J. W., D. E. Scott, T. J. Ryan, K. A. Buhlmann, T. D. Tuberville, B. S. Metts, J. L. Greene, T. Mills, Y. Leiden, S. Poppy y C. T. Winne. 2000. The global decline of reptiles, déja vu amphibians. BioScience 50:653-666.

González-Hernández, A. J. y J. M. Garza-Castro. 2006. 
Herpetofauna del municipio de Nuevo Urecho, Michoacán, México. In Inventarios herpetofaunísticos de México: avances en el conocimiento de su biodiversidad, A. RamírezBautista, L. Canseco-Márquez y F. Mendoza-Quijano (eds.). Publicaciones de la Sociedad Herpetológica Mexicana 3:140-151

Huacuz, E. D. 1995. Serpientes del estado de Michoacán. Tesis, Maestría Facultad de Ciencias, UNAM, México, D. F. 175 p.

INEGI (Instituto Nacional de Estadística Geografía e Informática). 1985. Síntesis geográfica del estado de Michoacán, Aguascalientes, Aguascalientes 315 p.

Lips, K. R., J. K. Reaser, B. E. Young y R. Ibañez. 2001. Amphibian monitoring in Latin America: a protocol manual/ Monitoreo de anfibios en América Latina: manual de protocolos. Herpetological Circular 30. Society for the Study of Amphibians and Reptiles [SSAR], Oxford, Ohio. 115 p.

Magurran, A. E. 2004. Measuring biological diversity. Blackwell Science, Malden, Massachusetts. 256 p.

Mittermeier, R. A., C. Goettsch y P. Robles-Gil. 1997. Megadiversidad: los países biológicamente más ricos del mundo. CEMEX, México, D. F. 501 p.

Mendelson, J. R. III., K. R. Lips, R. W. Gagliardo, G. B. Rabb, J. P. Collins, J. E. Diffendorfer, P. Daszak, R. Ibañez D., K. C. Zippel, D. P. Lawson, K. M. Wright, S. N. Stuart, C. Gascon, H. R. da Silva, P. A. Burrowes, R. L. Joglar, E. La Marca, S. Loters, L. H. du Preez, C. Weldon, A. Hyatt, J. V. RodríguezMahecha, S. Hunt, H. Robertson, B. Löck, C. J. Raxworthy, D. R. Frost, R. C. Lacy, R. A. Alford, J. A. Campbell, G. Parra-Olea, F. Bolaños, J. J. Calvo D., T. Halliday, J. B. Murphy, M. H. Wake, L. A. Coloma, S. L. Kuzmin, M. S. Price, K. H. Howell, M Lau, R. Pethiyagoda, M. Boone, A. R. Blaustein, A. Dobson, R. A. Griffiths, M. L. Crump, D. B. Wake y E. D. Brodie Jr. 2006. Confronting amphibian declines and extinctions. Science 313:48.

Ortega-Gómez, A. 2006. Distribución y propuesta de áreas prioritarias para la conservación del género Ambystoma en Michoacán. Tesis Maestría, Facultad de Biología, Universidad Michoacana de San Nicolás de Hidalgo, Morelia, Michoacán. 81 p.

Peters, A. J. 1954. The amphibians and reptiles of the coast and coastal sierra of Michoacán, México. Occasional Papers of the Museum of Zoology. University of Michigan 544:1-37.

Ramírez-Bautista, A. 1994. Manual y claves ilustradas de los anfibios y reptiles de la región de Chamela, Jalisco, México. Cuadernos del Instituto de Biología 23. Universidad Nacional Autónoma de México, México, D. F. 127 p.
Reyna, A. Y. 2010. Anfibios y reptiles del municipio de Huetamo, Michoacán, México. Tesis, Facultad de Biología, Universidad Michoacana de San Nicolás de Hidalgo, Morelia, Michoacán. $171 \mathrm{p}$.

Rodríguez, P., J. Soberón y H. T. Arita. 2003. El componente beta de la diversidad de mamíferos de México. Acta Zoológica Mexicana, nueva serie 89:241-259.

Schmidth, K. P. y F. A. Shannon. 1947. Notes on the amphibians and reptiles of Michoacán, México. Fieldiana Zoology 31:63-85.

Savage, J. 2002. The amphibians and reptiles of Costa Rica: A herpetofauna between two continents, between two seas. The University of Chicago Press, Illinois. 934 p.

SEMARNAT (Secretaría del Medio Ambiente y Recursos Naturales). 2002. Norma Oficial Mexicana NOM-059SEMARNAT-2001, Protección ambiental-Especies nativas de México de flora y fauna silvestres-Categorías de riesgo y especificaciones para su inclusión, exclusión o cambio-Lista de especies en riesgo. Diario Oficial de la Federación, 6 de marzo, México, D. F.

Smith, H. M. y E. H. Taylor. 1945. An annotated checklist and key to the snakes of Mexico. United States National Museum, Bulletin 187:1-239.

Smith, H. M. y E. H. Taylor. 1948. An annotated checklist and key to the amphibians of Mexico. United States National Museum, Bulletin 194:1-118.

Smith, H. M. y E. H. Taylor. 1950. An annotated checklist and key to the reptiles of Mexico exclusive of the snakes. United States National Museum, Bulletin 199:1-253.

Soberón, J. y J. Llorente. 1993. The use of species accumulation functions for the prediction of species richness. Conservation Biology 7:480-488.

Sokal, R. R. y F. J. Rohlf. 1985. Biometry. The principles and practice of statistics in biological research, tercera edición. W. H. Freeman, New York. 887 p.

Vargas-Santa María, F. y O. Flores-Villela. 2006. Estudio herpetofaunístico en el Playón de Mexiquillo y áreas adyacentes en la costa sur del estado de Michoacán, México. In Inventarios herpetofaunísticos de México: avances en el conocimiento de su biodiversidad, A. Ramírez-Bautista, L. Canseco-Márquez y F. Mendoza-Quijano (eds.). Publicaciones de la Sociedad Herpetológica Mexicana 3:110-136.

Vázquez, D. J. y G. E. Quintero. 2005. Anfibios y reptiles de Aguascalientes. Comisión Nacional para el Conocimiento y Uso de la Biodiversidad. México, D. F. 318 p. 
Apéndice 1. Lista de especies de anfibios y reptiles del municipio de Tacámbaro, Michoacán, México. Tipo de vegetación: BO, bosque de oyamel; BP, bosque de pino; BPE, bosque de pino encino; BE, bosque de encino; BES, bosque espinoso y SBC, selva baja caducifolia. Hábito: te, terrestre; ac, acuático; ar, arborícola; sax, saxícola; fos, fosorial; ri, ripario. Endemismo: EM, endémico de México; EMic, endémico de Michoacán. NOM-059 (NOM-59- SEMARNAT-2001). Categoría de riesgo: Pr, sujeta a protección especial, A, amenazada. * Registro nuevo para Michoacán.

\section{Taxa}

Clase Amphibia

Orden Caudata

Familia Ambystomatidae

Ambystoma ordinarium (Taylor, 1940)

Familia Plethodontidae

Pseudoeurycea belli (Gray, 1850)

Orden Anura

Familia Bufonidae

Rhinella marina (Linnaeus, 1758)

Incilius marmoreus (Wiegmann, 1833)

Incilius perplexus (Taylor, 1943)

Familia Craugastoridae

Craugastor hobarsmithi (Taylor 1937)

Familia Eleutherodactylidae

Eleutherodactylus nitidus (Peters, 1870)

Familia Hylidae

Exerodonta smaragdina (Taylor, 1940)

Hyla arenicolor (Cope 1866

Hyla eximia (Baird, 1854)

Hyla plicata (Brocchi, 1877)

Pachymedusa dacnicolor (Cope, 1864)

Plectrohyla bistincta (Cope, 1877)

Smilisca baudinii (Duméril y Bribon, 1841)

Tlalocohyla smithii (Boulenger, 1902)

Familia Leptodactylidae

Leptodactylus fragilis (Brochii,1877)

Leptodactylus melanonotus (Hallowell, 1861)

Familia Microhylidae

Hypopachus variolosus (Cope, 1866)

Familia Ranidae

Lithobates forreri (Boulenger, 1883)

Lithobates montezumae (Baird, 1854)

Lithobates zweifeli (Hillis, Frost y Weed, 1984)

Clase Reptilia

Orden Testudines

Familia Kinosternidae

Kinosternon hirtipes (Gagler, 1830)

Kinosternon integrum (Le Conte, 1854)

Orden Squamata

Suborden Lacertilia

Familia Anguidae

\section{Tipo de}

vegetación

BP, BPE

BO, BP

BE, BES, SBC
BES, SBC

$\mathrm{SBC}$

BP, BPE

BES, SBC

$\mathrm{BE}, \mathrm{BES}, \mathrm{SBC}$

$\mathrm{BP}, \mathrm{BES}, \mathrm{SBC}$

BPE, BE

BO

BES, SBC

$\mathrm{BP}, \mathrm{BE}$

$\mathrm{BE}, \mathrm{BES}, \mathrm{SBC}$

$\mathrm{BE}, \mathrm{BES}, \mathrm{SBC}$

BES, SBC

BES

BE, BES

$\mathrm{BE}, \mathrm{BES}, \mathrm{SBC}$

BPE

SBC

BE

BES, SBC

Hábito

Endemismo

NOM-059

(n)


Apéndice 1. Continúa

\section{Taxa}

Barisia imbricata (Wiegmann, 1828)

Familia Corytophanidae

Basiliscus vittatus (Wiegmann, 1828)

Familia Eublepharidae

Coleonyx elegans (Gray, 1845)

Familia Gekkonidae

Hemidactylus frenatus (Dumeril y Bibron, 1836)

Phyllodactylus lanei (Smith, 1935)

Familia Helodermatidae

Heloderma horridum (Wiegmann, 1829)

Familia Iguanidae

Ctenosaura pectinata (Wiegmann, 1834)

Familia Phrynosomatidae

Sceloporus dugesi (Bocourt, 1873)

Sceloporus gadoviae (Boulenger, 1905)

Sceloporus grammicus (Wiegmann, 1928)

Sceloporus horridus (Wiegmann, 1834)

Sceloporus melanorhinus (Bocourt, 1876)

Sceloporus pyrocephalus (Cope, 1864)

Sceloporus scalaris (Wiegmann, 1828)

Sceloporus torquatus (Wiegmann, 1828)

Sceloporus utiformis (Cope, 1864)

Urosaurus bicarinatus (Duméril, 1856)

Familia Polycrhotidae

Anolis nebulosus (Wiegmann, 1834)

Familia Scincidae

Mesoscincus altamirani (Dugés, 1891)

Plestiodon copei (Taylor, 1933)

Plestiodon dugesi (Thominot, 1883)

Familia Teiidae

Ameiva undulata (Wiegman, 1834)

Aspidocelis communis (Cope, 1878)

Suborden Serpentes

Familia Boidae

Boa constrictor (Linnaeus, 1758)

Familia Colubridae

Conopsis biserialis (Taylor y Smith, 1942)

Conopsis nasus (Günther, 1858)

Drymarchon melanurus (Suméril, Bibron y Duméril, 1854)

Drymobius margaritiferus (Schlegel, 1837)

Enulius flavitorques (Cope, 1869)

Imantodes gemmistratus (Cope, 1861)

Lampropeltis triangulum (Lacépéde, 1789)

Leptodeira maculata (Hallowell, 1861)

Leptodeira splendida (Günter, 1895)

\section{Tipo de vegetación}

BPE

BES, SBC

$\mathrm{SBC}$

$\mathrm{SBC}$

BES,SBC

BES

BES,SBC

$\mathrm{BP}, \mathrm{BPE}$

SBC

BO,BP, BPE

$\mathrm{BP}, \mathrm{BE}, \mathrm{BES}, \mathrm{SBC}$

BES, SBC

BES,SBC

BO

$\mathrm{BP}, \mathrm{BPE}$

BE

BP, SBC

BP, BPE, SBC

SBC

BO

BPE

SBC

BES, SBC

BES, SBC

BPE

BPE

SBC

BES, SBC

SBC

$\mathrm{BE}$

BE

BES, SBC

SBC
Hábito

Te

Ri

$\mathrm{Te}$

$\mathrm{Ar}$

Te, Ar, Sax

$\mathrm{Te}$

Te, Ar, Sax

Te, Ar, Sax

Ar

Sax

Te

Ar, Sax

$\mathrm{Te}$

Ar

$\mathrm{Te}, \mathrm{Ar}$

$\mathrm{Te}$

$\mathrm{Te}$

$\mathrm{Te}$

$\mathrm{Te}, \mathrm{Ri}$

EM

EM

Fos

$\mathrm{Te}, \mathrm{Ri}$

$\mathrm{Te}$

$\mathrm{Te}$

Te

Te, Ri, Ar

Te, Ri
A

A

$\operatorname{Pr}$

EM

Pr

A

EM

A

Pr

A

A

r

EM

EM

EM

EM

EM

EM $\quad \mathrm{Pr}$

$\mathrm{Pr}$

A

Pr


Apéndice 1. Continúa

\section{Taxa}

Leptophis diplotropis (Günther, 1872)

Masticophis mentovarius (Duméril, Bibron y Duméril, 1854)

Oxybelis aeneus (Wagler, 1824)

Pituophis lineaticollis (Cope, 1861)

Pseudoleptodeira latifasciata (Günther, 1894)

Rhadinaea hesperia (Bailey, 1940)

Salvadora mexicana (Duméril, Bibron y Duméril, 1854)

Senticolis triaspis (Cope, 1866)

Sonora michoacanensis (Dugés, 1884)

Storeria storerioides (Cope, 1865)

Tantilla bocourti (Günther, 1895)

Tantilla calamarina (Cope, 1866)

Thamnophis cyrtopsis (Kennicott, 1860)

Thamnophis scalaris (Cope, 1861)

Trimorphodon biscutatus (Duméril, Bibron y Duméril, 1854)

Trimorphodon tau (Cope, 1870)

Familia Leptotyphlopidae

Leptotyphlops bressoni (Taylor, 1939)

* Leptotyphlops maximus (Loveridge,1932)

Familia Typhlopidae

Ramphotyphlops bramminus (Daudin, 1803)

Familia Viperidae

Agkistrodon bilineatus (Günther, 1863)

Crotalus simus (Klauber, 1952)

Crotalus molossus (Baird y Girard, 1853)

Crotalus triseriatus (Wagler, 1830)

\begin{tabular}{|c|c|c|c|}
\hline $\begin{array}{c}\text { Tipo de } \\
\text { vegetación }\end{array}$ & Hábito & Endemismo & $N O M-059$ \\
\hline $\mathrm{SBC}$ & $\mathrm{Ar}$ & EM & A \\
\hline $\mathrm{SBC}$ & $\mathrm{Te}$ & EM & A \\
\hline $\mathrm{SBC}$ & $\mathrm{Ar}$ & & \\
\hline BPE & $\mathrm{Te}$ & EM & \\
\hline $\mathrm{SBC}$ & $\mathrm{Ar}$ & EM & $\operatorname{Pr}$ \\
\hline BES & $\mathrm{Te}$ & EM & $\operatorname{Pr}$ \\
\hline $\mathrm{SBC}$ & $\mathrm{Te}$ & EM & $\operatorname{Pr}$ \\
\hline $\mathrm{SBC}$ & $\mathrm{Te}$ & EM & \\
\hline BES & Fos & EM & \\
\hline BPE & $\mathrm{Te}$ & EM & \\
\hline BES & Fos & EM & \\
\hline BES, SBC & Fos & EM & $\operatorname{Pr}$ \\
\hline BP & $\mathrm{Te}$ & & A \\
\hline во & $\mathrm{Te}$ & EM & A \\
\hline $\mathrm{SBC}$ & $\mathrm{Te}$ & & \\
\hline $\mathrm{SBC}$ & $\mathrm{Te}$ & EM & \\
\hline $\mathrm{SBC}$ & $\mathrm{Te}$ & EMic & $\operatorname{Pr}$ \\
\hline $\mathrm{SBC}$ & $\mathrm{Te}$ & EM & \\
\hline BES & $\mathrm{Te}$ & & \\
\hline BES & $\mathrm{Te}$ & & \\
\hline BES & $\mathrm{Te}$ & & \\
\hline BPE & $\mathrm{Te}$ & & $\operatorname{Pr}$ \\
\hline BO, ВР, ВРЕ & $\mathrm{Te}$ & EM & \\
\hline
\end{tabular}

